\title{
Face Recognition in Young Children: When the Whole is Greater than the Sum of Its Parts
}

\author{
James W. Tanaka, Joshua B. Kay, Eliza Grinnell, Brent Stansfield \\ and Lisa Szechter \\ Department of Psychology, Oberlin College, Oberlin, OH, USA
}

\begin{abstract}
Do young children recognize faces differently than older children and adults? Previous research (Carey \& Diamond, 1977) has suggested that, before the age of 8 , children recognize a face by its individual features; after the age of 8 , they switch to a whole-face (holistic) recognition strategy. The part-whole paradigm provides a suitable test for the encoding switch hypothesis. In this paradigm, memory for a face part is probed when the part is presented in isolation and in the whole face. The difference in performance between the two test conditions serves as an index of holistic processing. Results from such studies reveal that even 6-year-olds remember parts from upright faces better when tested in the whole-face context than in isolation. When faces are inverted, the holistic processes of young children and older children are disrupted. These results indicate that counter to the encoding switch hypothesis, children recognize faces holistically by 6 years of age.
\end{abstract}

\section{INTRODUCTION}

Although recent studies indicate that humans are born with an innate preference for faces (Johnson \& Morton, 1991), normal face processing abilities are not fully developed until about the age of 12 years. The development of face processing abilities has clearly been shown in studies in which older children (12-year-olds) performed better than younger children (6-year-olds) on tasks involving face perception (Ellis, 1992; Saltz \& Siegel, 1967) and face recognition (Carey, Diamond, \& Woods, 1980). While there is little question that face

Requests for reprints should be addressed to James W. Tanaka, Department of Psychology, Severance Laboratory, Oberlin College, Oberlin, OH44074, USA. E-mail: tanaka@ occs.cs.oberlin.edu

We are grateful to Glen Humphreys, Sue Carey and two anonymous reviewers for their valuable comments. We would also like to thank the children and teachers of Eastwood and Prospect Elementary Schools for their help and cooperation. This research as supported by NIH grant R15 HD30433 and a Keck Foundation Faculty Research Award. 
processing abilities improve with age, there is much debate as to the underlying processes that might account for these age-related differences in performance. At issue is whether young children lack some basic process associated with mature face processing or whether their face processes are simply not as refined as older children's and adults' face processes.

Some empirical evidence suggests that there are fundamental differences between the way young children and older children perceive and remember faces. In the adult face recognition literature, one of the most robust findings is that inversion disproportionately impairs the recognition of faces more than the recognition of other objects (e.g. houses, aeroplanes, stick figures) (Diamond \& Carey, 1986; Yin, 1969). Indeed, the face inversion effect has led some researchers to claim that face recognition is somehow special compared to other types of object recognition (Yin, 1969). Given the robustness of the face inversion effect in adults, it was surprising to find that young children failed to exhibit a similar effect of orientation when recognizing faces. In a seminal study, Carey and Diamond (1977) showed 6-, 8- and 10-year-old children upright and inverted photographs of unfamiliar people. Immediately after seeing the photographs, they were asked to identify the previously seen "old" face from a never-before-seen "new" face. Carey and Diamond found that 8and 10-year-olds, like adults, recognized the "old" faces better when they were shown in their upright orientation than when shown in the inverted orientation. However, the 6-year-olds recognized the inverted faces equally as well as the upright faces. The finding that 6-year-olds performed similarly across the two orientations led Carey and Diamond to hypothesize that children of this age use a featural encoding strategy for processing upright and inverted faces. They proposed an "encoding switch hypothesis" in which children, at least until 6 years of age, encode upright faces according to their constituent features (e.g. eyes, nose, mouth), and then, around the age of 10 , begin to process faces holistically.

Carey and Diamond (1977) tested this hypothesis in a second experiment in which children identified faces across changes in paraphernalia (e.g. clothing, hairstyle and eyeglasses) and facial expression. Their prediction was that, if young children attend more to isolable featural information in faces, they would be more likely than older children to make false recognition decisions based on paraphernalia and facial expression. Consistent with their prediction, they found that the 6-year-olds were misled more by changes in paraphernalia and facial expressions than 8- and 10-year-olds. Thus, drawing on evidence from their inversion and paraphernalia studies, Carey and Diamond claimed that children initially process faces according to their parts and then, by the age of 10, switch from a piecemeal encoding approach to a holistic approach.

Other researchers have challenged Carey and Diamond's (1977) encoding switch claims (Flin, 1985; Valentine, 1988). Flin argued that the recognition task used in Carey and Diamond's study might have been too demanding for 
the 6-year-olds and, even though they responded above chance levels, their generally poor performance might have obscured possible inversion effects. To avoid floor effects, Flin used an "old/new" recognition task in which inversion effects were measured as differences in $d^{\prime}$ between upright and inverted face test conditions. Consistent with Carey and Diamond, Flin found that overall recognition of the 6-year-olds was below the level obtained by the older groups. However, in contrast to Carey and Diamond, Flin found that the magnitude of the $d^{\prime}$ differences in the upright and inverted conditions was the same for the 6-year-olds as for the 8- and 10-year-olds. Flin argued that, when age-related performance differences are taken into account, there is little evidence to support the encoding switch hypothesis.

However, Carey and Diamond (1994) have recently demonstrated that inversion effects do increase as a function of age when reaction time rather than accuracy is used as the relevant dependent measure. Following a composite face recognition paradigm developed by Young, Hellawell and Hay (1987), 6-year-old, 10-year-old and adult participants were asked to identify composite and non-composite photographs of their classmates when presented in their upright and inverted orientations. The composite photographs were constructed by aligning the top half of a classmate's face with the bottom half of another. In the composite face conditions, the children were instructed to identify the classmate depicted in the top half of the photograph. Carey and Diamond found that the 6-year-olds, like the 10-year-olds and the adults, were slower to recognize composite faces than the non-composite faces when shown in their upright orientation. When the composite and non-composite faces were inverted, the inverted composite faces were recognized as quickly as their non-composite versions. However, the younger children demonstrated no inversion effect in that they were equally fast to name inverted faces as upright faces. In marked contrast, the older children and adults were slower to name the inverted faces relative to the upright faces. Thus, the presence and magnitude of the inversion effect, as measured by naming latencies, increased as a function of age.

Although face inversion studies reveal performance differences between younger and older children, they provide little insight into the cognitive operations that might produce these differences. As described earlier, Carey and Diamond offered one account for these differences: They believed that upright faces are processed holistically, whereas inverted faces are processed featurally. Unfortunately, without operational descriptions of "holistic" and "featural", this distinction is not directly testable. Recently, Tanaka and Farah (1993) operationalized the concept of holistic and featural processing in the following task: Participants were shown either an upright or inverted target face accompanied by a verbally spoken name. At test, participants identified parts of the target face (e.g. Joe's nose) from its foil when presented in isolation or in the context of the whole face (see Figure 1). Importantly, in the whole-face 


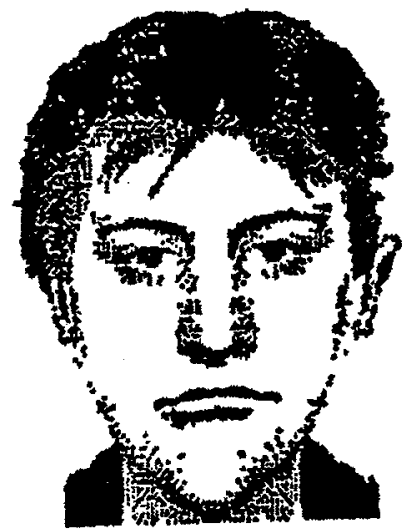

Larry
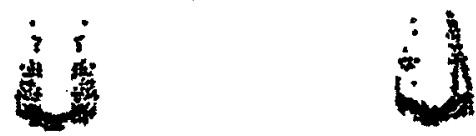

Which is Larry's Nose?
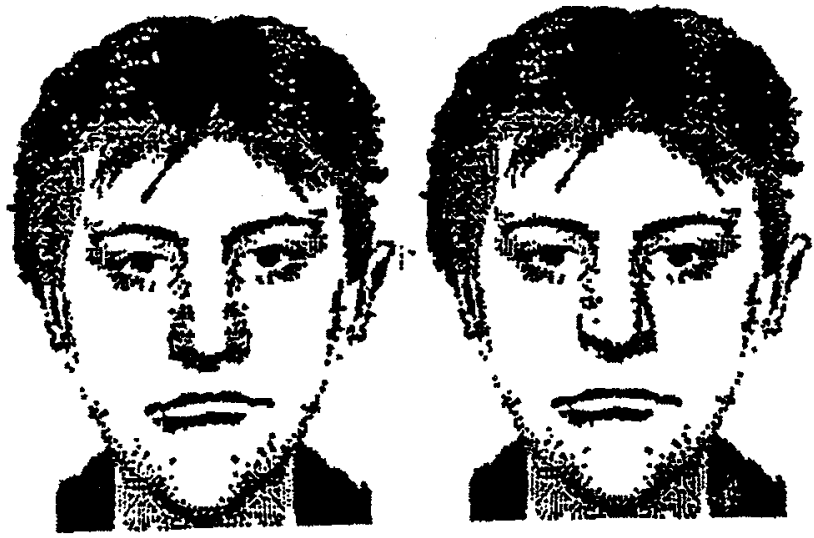

\section{Which is Larry?}

FIG. 1. Example of part-whole test items. In this example, after the participants learnedLarry's face, their memory for Larry's nose was tested when it was presented in isolation and in the whole face. Note that, in the whole-face condition, the two faces differed only with respect to the nose feature.

test condition, the target and foil faces only differed according to the target feature under test - the other features of the face were exactly the same. Tanaka and Farah reasoned that, if upright faces are encoded holistically, the wholeface test item should serve as a better retrieval cue than the isolated-part test item. In contrast, if inverted faces are encoded only in terms of their parts, there 
should be no difference in the isolated-part and whole-face test conditions. Consistent with a holistic theory of face recognition, they found that face parts from upright faces were more easily recognized in the context of the whole face than in isolation. Crucially, there was no difference in the recognition of parts from inverted faces whether the parts were presented in isolation or in the whole-face context. According to the holistic hypothesis, normal, upright faces are processed holistically, whereas inverted faces are processed featurally (see Farah, Drain, \& Tanaka, 1995).

The value of the part-whole paradigm is that it provides a direct means of testing the encoding switch hypothesis. In the following experiments, 6-, 8- and 10 -year-old children learned to identify a series of upright faces by name. After learning, their memory for the face parts was tested when the parts were presented in isolation and in the whole face. According to the encoding switch hypothesis, if young children process faces featurally, their recognition of face parts should be the same whether the parts are tested in isolation or in the whole face. On the other hand, if young children, like adults, rely on a holistic encoding strategy, their recognition of the parts should be reliably better in the whole-face condition relative to the isolated-part condition. In Experiment 1, to avoid the possibility contamination due to floor effects, the children practised identifying the face stimuli until they were able to recognize all faces without error.

\section{EXPERIMENT 1}

\section{Method}

Participants. Twenty-four first-grade children (mean age $=6.4$ years), 24 third-grade children (mean age $=8.75$ years) and 24 fifth-grade children (mean age $=10.56$ years) enrolled in the Oberlin school district participated in this study. Thus, a total of 72 children participated in Experiment 1.

Materials. The face stimuli consisted of four male faces generated on a Macintosh computer using the Mac-a-Mug program. The faces were constructed such that each face had a unique set of eyes, nose and face features placed in the same face outline (similar to the ones shown in Figure 1). A panel of three judges rated the faces as highly discriminable from each other. The stimuli measured $2 \times 3$ inches in size and were photocopied onto white card stock.

Procedure. The participants were tested individually. Each child was seated at a table directly facing the experimenter at a viewing distance of approximately $1 \mathrm{~m}$.

In the learning phase, the children were told that they were going to play a matching game in which they would see four faces and their task was to learn 
the name that matched the face. The names of the four faces (i.e. "Tom", "Bob", "Joe" and "Fred") were printed in 18 point Helvetica bold type font onto a white index card and placed in a row in front of the children. To familiarize the children with the faces, the experimenter randomly presented each face two times and pointed to the name card that corresponded to the face. After familiarization, each child was shown a face and asked to name the face. If the child responded with the incorrect name or could give no response, the experimenter pointed to the correct name label and went on to the next face in the learning block. A learning block consisted of two presentations of each of the four faces. The faces were presented at random within a learning block with the restriction that the same face was not shown on consecutive learning trials. Training continued until the child identified all faces in the learning block without error.

In the test phase, immediately following learning, a two-choice recognition test was administered. In the isolated feature condition, the children saw a target and foil exemplar presented in isolation. They were asked to identify the correct target exemplar (e.g. "Tom's nose") and were given unlimited time to make their selection. If the children were uncertain of their response, they were instructed to make their best guess. In the equivalent whole-face condition, the children saw the target and foil exemplars embedded in a whole face and were asked to identify the correct target face. The whole-face stimuli were constructed such that they differed only with respect to the critical feature under test; the other features were held constant. The left and right positions of the correct isolated-part and whole-face target stimuli were counterbalanced across test items. The three features (eyes, nose and mouth), two test conditions (isolated part $v s$ whole face) and four faces yielded a total of 24 experimental trials. The experimental trials were presented at random with the restriction that items from the same face could not be tested on consecutive trials.

Finally, in the post-test phase, the children's memory for the four faces was assessed. The experimenter presented each face two times and the child identified the face by pointing to the name card. The faces were randomly presented with the restriction that the same face did not occur on consecutive trials.

\section{Results and Discussion}

Pre- and Post-test Performance. As expected, the younger children required more learning blocks to reach criterion than the older children. The 6-year-olds needed 5.17 blocks to achieve criterion, the 8-year-olds needed 3.92 blocks and the 10-year-olds needed 2.79 blocks. An analysis of variance with age as the between-groups factor confirmed these training differences, $F(2,69)$ $=4.72, M S_{\mathrm{e}}=33.88, p<.01$. 
As indicated by their post-test performance, the older children remembered the faces better than the younger children. The 10-year-olds correctly identified 7.54 faces in the post-test compared with 7.00 faces identified by the 8-yearolds and 6.46 faces by the 6 -year-olds. The post-test differences were confirmed in a one-way analysis of variance with age as the between-groups factor: $F(2,69)=4.05, M S_{\mathrm{e}}=7.04, p<.02$.

Part-Whole Test Performance. Overall, the children recognized face parts better when presented in the whole face than when presented in isolation. As shown in Figure 2, the 6-year-olds correctly identified face parts presented in isolation on $58 \%$ of the trials compared with $67 \%$ when embedded in the whole face. The 8 -year-olds correctly identified face parts on $62 \%$ of trials in the isolated condition and $77 \%$ in the whole-face condition. The 10 -year-olds correctly identified face parts on $71 \%$ of trials in the isolated condition and $81 \%$ in the whole-face condition. An analysis of variance with test condition (isolated part $v s$ whole face) as a within-participant factor and age ( 6,8 and 10 years of age) as a between-groups factor confirmed the main effect of test condition, $F(1,69)=27.82, M S_{\mathrm{e}}=66.69, p<.01$. Direct comparisons revealed that all three age groups recognized face parts better in the whole-face condition than in the isolated condition, $p<.05$.

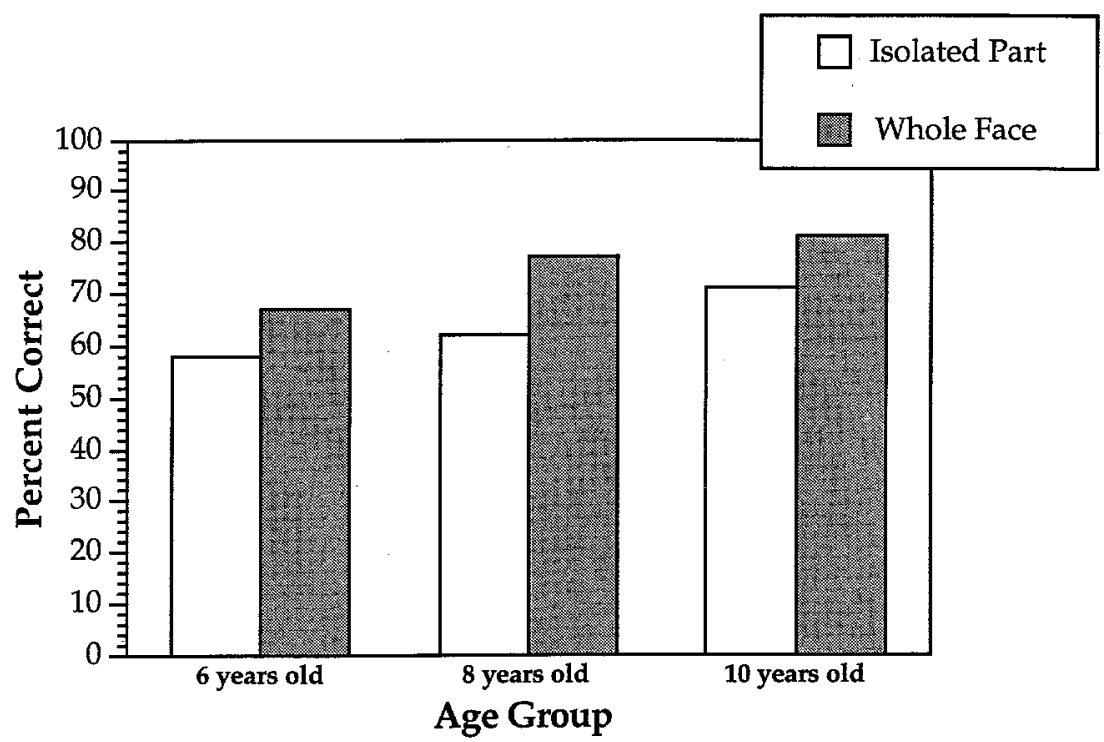

FIG. 2. Results of Experiment 1. Percentage of correctly identified isolated-part and whole-face test items as a function of age. 
The main effect of age was also significant, $F(2,69)=9.39, M S_{\mathrm{e}}=30.11, p$ $<.001$. Overall, older children correctly identified more face parts than younger children whether the parts were presented in isolation or in the whole face. However, the critical interaction between test condition and age was not significant, $F(2,69)=0.60, M S_{\mathrm{e}}=1.44, p<.10$. Although the children's overall recognition improved with age, the difference between their performance in the isolated and whole-face test conditions remained relatively constant across the three age groups.

\section{EXPERIMENT 2}

In Experiment 1, we found that, by the age of 6 years, children formed holistic representations of faces as manifested by their improved ability to recognize face parts presented in the whole face than in isolation. Hence, these results failed to support the prediction of the encoding switch hypothesis. One difference between Experiment 1 and Carey and Diamond's (1977) experiment was that, in the part-whole task, participants were required to remember the target faces over a fairly long retention interval, whereas in Carey and Diamond's study, recognition was tested immediately following inspection. It may be that long-term memory tasks involve the formation of holistic face representations even in young children, whereas immediate memory tasks allow for featural processing. To test this possibility, we modified the part-whole paradigm to be an immediate memory task. In this study, children inspected a target face for 5 sec and then, immediately following inspection, the children identified a face part presented in isolation and in the whole face. If young children rely on featural information to encode faces in immediate memory, we would expect the difference in their "parts" and "wholes" performance to be less than the part-whole difference of older children. On the other hand, if young children form holistic representations in immediate memory, then we would expect the magnitude or part-whole differences to be the same across age groups.

\section{Method}

Participants. Twenty-four first-grade children (mean age $=6.6$ years), second-grade children (mean age $=7.8$ years), fourth-grade children (mean age $=9.5$ years) and sixth-grade children (mean age $=11.3$ years) from the Oberlin school district participated in this study. Thus, a total of 96 children participated in Experiment 2.

Materials. The stimuli for this study were taken from 28 black and white photographs of 6-year-olds. The photographs were digitized into a Macintosh computer with a MicroTek Z scanner. Four composite faces were generated with the Adobe Photoshop graphics package using the eyes of one child, the 
nose of a second child, the mouth of a third child and the face outline of another child (see Figure 3 ). Two of the composite faces were of boys and two were of girls. In addition to the four composite target faces, composite foil faces were generated by replacing either the eye, nose or mouth feature of the target face with the eye, nose or mouth feature from a new face. Isolated-part versions of target and foil features were made by removing the feature from the face and placing it on a white background. Hard copy versions of the whole-face and part-face stimuli were printed and formatted onto $4 \times 6$ inch index cards. The left and right locations of the target stimulus were counterbalanced across test items.

Procedure. The children were told that they would be playing a matching game. The experimenter presented the target face and identified it by name (e.g. "This is Bill"). The children were given $5 \mathrm{sec}$ to inspect the face. Immediately following inspection, in the whole-face test condition, children saw the target face and the foil face that differed from the target by one feature. Participants were asked to point to the face that they had just seen (e.g. "Which is Bill?"). In the isolated-part test condition, children saw the target feature and the foil feature and were asked to point to the feature that they had just seen (e.g. "Which is Bill's nose?"). The left and right positions of the isolated-part and full-face target stimuli were counterbalanced. The three features (eyes, nose and mouth), two test conditions (isolated part $v s$ whole face) and four faces yielded a total of 24 experimental trials. Experimental trials were randomly presented with the restriction that items from the same face could not be tested on consecutive trials.
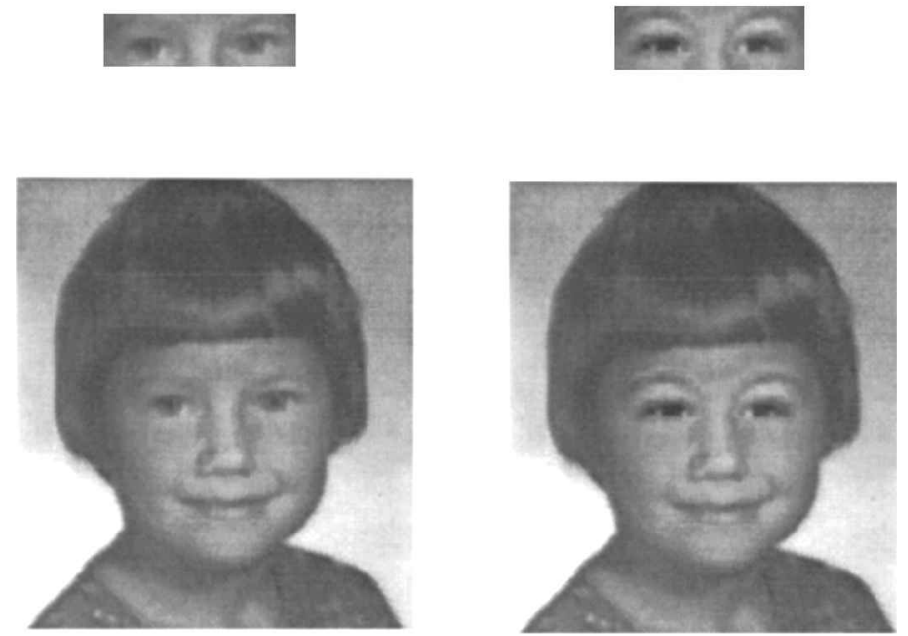

FIG. 3. Example of composite face images used in Experiment 2 for the isolated-part and whole-face tests. 


\section{Results and Discussion}

As shown in Figure 4, children at all age levels were significantly better at recognizing parts presented in whole faces than presented in isolation. The 6-year-olds recognized $62 \%$ of the parts in isolation, compared with $73 \%$ in the whole face; the 7-year-olds recognized $61 \%$ in isolation and $80 \%$ in the whole face; the 9 -year-olds recognized $75 \%$ in isolation and $86 \%$ in the whole face; and the 11 -year-olds recognized $77 \%$ in isolation and $85 \%$ in the whole face. An analysis of variance with test condition (isolated part $v s$ whole face) as a within-participants factor and age $(6,7,9$ and 11 years of age) as a betweengroups factor confirmed the main effect of test condition, $F(1,88)=54.92, M S_{\text {e }}$ $=34.71, p<.01$. Direct comparisons revealed that all four age groups recognized face parts better in the whole-face condition than in the isolated-part condition, $p<.05$. The other main effect of age was also significant, $F(3,88)=$ $8.83, M S_{\mathrm{e}}=11.42, p<.01$, demonstrating that older children performed better than the younger children in the recognition task. However, the critical interaction between test condition and age was not significant, $F(1,3)=1.89, M S_{\text {e }}$ $=1.19, p<.10$. Consistent with the results of Experiment 1, the relative difference with which children were better at identifying the part in the whole face than in isolation did not change across the four age groups. Hence, there was no evidence to suggest that children switched from a piecemeal encoding strategy to a holistic strategy as a function of age.

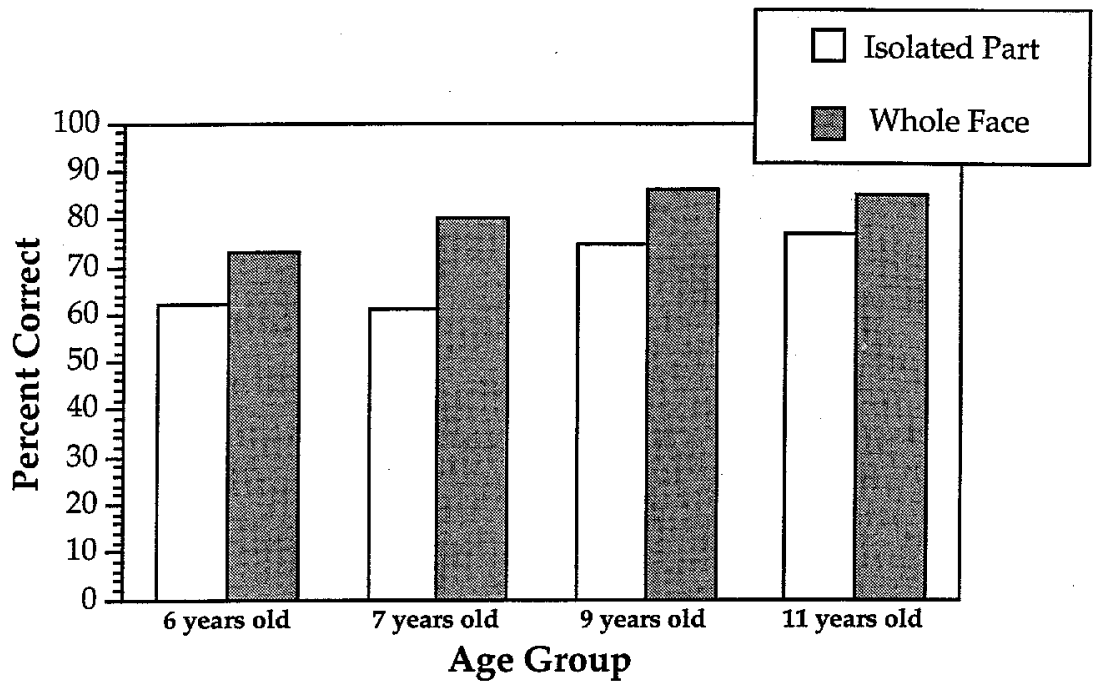

FIG. 4. Results of Experiment 2. Percentage of correctly identified isolated-part and whole-face test items as a function of age. 
In summary, the hypothesis that, in an immediate memory task of face recognition, younger and older children might rely on different featural and holistic strategies was not supported by the results of Experiment 2 .

\section{EXPERIMENT 3}

The results of Experiments 1 and 2 indicate that very young children remember upright faces holistically. However, the foregoing studies did not address how young (and older) children process inverted faces. Carey and Diamond (1977) speculated that children and adults recognize inverted faces on the basis of individual features. Consistent with this claim, Tanaka and Farah (1993) found that holistic recognition in adults is disrupted when a face is turned upsidedown. If young children, like adults, process inverted faces according to part rather than whole-face information, their holistic recognition should be similarly disrupted when faces are inverted. Alternatively, other research has shown that young children, in general, tend to adopt holistic strategies over featural or analytic strategies (Kemler Nelson, 1989). If this is the case, young children might demonstrate holistic recognition for inverted faces as well as upright faces.

\section{Method}

Participants. Twenty-five first-grade children (mean age $=6.8$ years), 27 third-grade children (mean age $=8.4$ years) and 16 fifth-grade children (mean age $=10.6$ years) enrolled in the Oberlin school district participated in this study. Thus, a total of 68 children participated in Experiment 3.

Materials. The stimuli for this study were taken from black and white photographs of 6- and 10-year-old children. The photographs were digitized into a Macintosh computer with a MicroTek Z scanner. Composite and foil composite faces were constructed according to the same procedure described in Experiment 2. Eight composite faces were generated; four faces were of boys and four were of girls. As in Experiment 2, isolated-part versions of target and foil features were made by removing the feature from the face and placing it on a white background. Hard copy versions of the full-face and part-face stimuli were printed and formatted onto $4 \times 6$ inch index cards. The left and right locations of the target stimulus were counterbalanced across test items.

Procedure. The procedure was identical to that of Experiment 2 except that eight target faces were used; four faces (two male and two female) were presented in an upright orientation, and four faces (two male and two female) were presented in an inverted orientation. In both the whole-face test condition and the isolated-part test condition, faces were presented in the same orientation 
as they had been introduced. For example, if the target face "Bill" was introduced in an upright orientation, the target and foil faces in the whole-face test condition, and the target and foil features in the isolated-part test condition were presented in an upright orientation. The orientation of the target faces was counterbalanced across subjects within each age group. As in Experiment 2, the left and right positions of the isolated-part and full-face target stimuli were counterbalanced. The three features (eyes, nose and mouth), two test conditions (isolated part $v s$ whole face) and eight faces yielded a total of 48 experimental trials. Experimental trials were presented at random with the restriction that items from the same face could not be tested on consecutive trials.

\section{Results}

Table 1 presents the performance of each age group in the whole-face and isolated-part test conditions in the upright and inverted orientations. An analysis of variance showed a main effect of test type, such that children recognized face parts better when presented in the whole face (mean $=64 \%)$ than when presented in isolation $($ mean $=56 \%), F(1,65)=21.88, M S_{\mathrm{e}}=65.48, p<.001$. An orientation $\times$ test type interaction, $F(1,65)=11.27, M S_{\mathrm{e}}=31.09, p<.01$, revealed that this whole-face advantage was present only in the upright orientation (see Figure 5a). Thus children were able to recognize upright face parts better when presented in the whole upright face $($ mean $=70 \%)$ than when presented in isolation (mean $=56 \%$ ), but there was no significant difference in their recognition of inverted face parts, whether presented in the whole inverted face $($ mean $=58 \%)$ or in isolation $($ mean $=56 \%$ ) (see Figure $5 \mathrm{~b})$.

A main effect of orientation was found, such that, overall, children were better able to recognize upright face parts, both in a whole face and in isolation $($ mean $=63 \%)$, than inverted face parts $($ mean $=57 \%), F(1,65)=18.25, M S_{\mathrm{e}}=$ 39.91, $p<.001$. An age $\times$ orientation interaction, $F(2,65)=3.22, M S_{\mathrm{e}}=7.04$, $p<.05$, revealed that the children's recognition of upright face parts varied as a function of their age, whereas their recognition of inverted face parts did not.

TABLE 1

Performance in the Whole-face and Isolated-part

Test Conditions in the Upright and Inverted

Orientations (Percentages)

\begin{tabular}{lccc}
\hline & 6-year-olds & 8-year-olds & 10-year-olds \\
\hline $\begin{array}{l}\text { Upright } \\
\text { Parts }\end{array}$ & 55 & 57 & 58 \\
Whole & 64 & 74 & 75 \\
& & & \\
Inverted & 59 & 53 & 55 \\
Parts & 57 & 57 & 62 \\
Whole & & & \\
\hline
\end{tabular}


The 10-year-old children recognized $66 \%$ of the upright face parts, both in a whole face and in isolation; the 8-year-old children recognized $65 \%$, and the 6 -year-old children recognized $60 \%$. In contrast, the 10 -year-old children recognized $59 \%$ of the inverted face parts, both in a whole face and in isolation; the 8 -year-old children recognized $55 \%$ and the 6-year-old children recognized $58 \%$. Thus, older children were better able to recognize upright face parts than younger children, but their recognition of inverted face parts did not vary significantly from that of the younger children. The age $\times$ orientation interaction suggests that better face recognition performance of older children cannot be due to their generally improved memory abilities. If this were the case, older children should have outperformed younger children in both the inverted and upright conditions. Because performance was restricted to the recognition of upright faces, the recognition advantage demonstrated by the older children seems to be related to their experience with upright faces. In the next section, we address the role of experience in the development of normal face recognition processes.

\section{GENERAL DISCUSSION}

The present study makes two points about face recognition development: First, by the age of 6 years, children are encoding faces holistically as shown by their better recognition of face parts presented in the whole face than in isolation. Second, the holistic advantage remains relatively stable from age 6 to age 10 . While the older children performed better than the younger children in both the isolated-part and whole-face test conditions, the relative difference between the two conditions did not vary with age. In summary, our results fail to support the claim that children switch from a featural encoding strategy to a holistic strategy as they grow older. Instead, it appears that, early on, children process faces holistically and the holistic advantage is maintained through adulthood.

How can the face inversion effects results be reconciled with our part-whole results? The critical finding for the encoding switch hypothesis was that 6-year-olds recognized inverted faces as well as upright faces. Assuming that inverted faces are encoded in a piecemeal fashion, the encoding switch hypothesis postulates that very young children are applying a similar featural strategy for recognizing upright faces. However, it does not necessarily follow that 6-year-olds are using the same encoding strategies simply because their recognition performance for the two types of stimuli were equivalent. Alternatively, our results suggest that, although younger children do not recognize upright faces as well as older children and adults, they nevertheless recognize faces holistically.

The recent results of Baenninger (1994, experiment 2) also support the holistic position. In her study, 8-year-old, 10-year-old and adult participants viewed an intact face; then, at test, they identified a scrambled version of the 
studied face. Scrambling the spatial locations of the eyes, nose and mouth features disrupts the configural information in the face. According to the encoding switch hypothesis, if young children encode faces in a piecemeal fashion, disrupting configural information should produce less interference for the 8 -year-olds than the 10 -year-olds or adults. However, counter to the prediction of the encoding switch hypothesis, configural disruption produced similar amounts of interference across the three age groups. Collectively, the present study, as well as the recent work of Carey and Diamond (1994) and Baenninger (1994), argue against the transitional view of face recognition development. Rather than viewing 5-year-old children as encoding a face on the basis of its features and then switching to a more configural encoding strategy by the age of 9 years, a growing body of evidence indicates that, by the age of 6 years, children are perceiving and remembering normal faces holistically.

The present results also address the seemingly discrepant evidence concerning face inversion and face composite effects in young children. Young children, unlike older children and adults, can recognize upside-down faces as well as upright faces (Carey \& Diamond, 1977). In contrast, similar to older children and adults, young children find it difficult to recognize a composite face when its top-half and bottom-half are aligned versus when they are misaligned (Carey \& Diamond, 1994). The opposing findings that young children fail to show a face inversion effect on the one hand, while demonstrating a face composite effect on the other, has led researchers to speculate that the two tasks are mediated by distinct cognitive processes (Carey \& Diamond, 1994). Specifically, it has been suggested that one recognition process, as indexed by the face inversion measure, emerges over time and is sensitive to the factors of age and experience. The other process, as indexed by the face composite measure, is either biologically endowed or acquired at a fairly early age (before age 6 years). However, the present results suggest an alternative explanation to the dual process account.

Inspection of whole-face performance in Figure 5 suggests that the emergence of an inversion effect with age hinges on two factors: First, the poor ability of young children to recognize whole upright faces relative to older children; second, the poor, but stable performance of all age groups to recognize the wholes of inverted faces. Thus, when the absolute levels of holistic performance are taken into account, young children show an absent or diminished inversion effect compared with older children. In contrast, when the relative holistic performance (i.e. the difference in part and whole recognition) is considered, young children show the same magnitude of holistic processing as older children and adults. Therefore, rather than postulating two separate processes, it is possible that different levels (absolute and relative) of the same holistic process might explain why young children demonstrate a face composite effect, but fail to show a face inversion effect. 

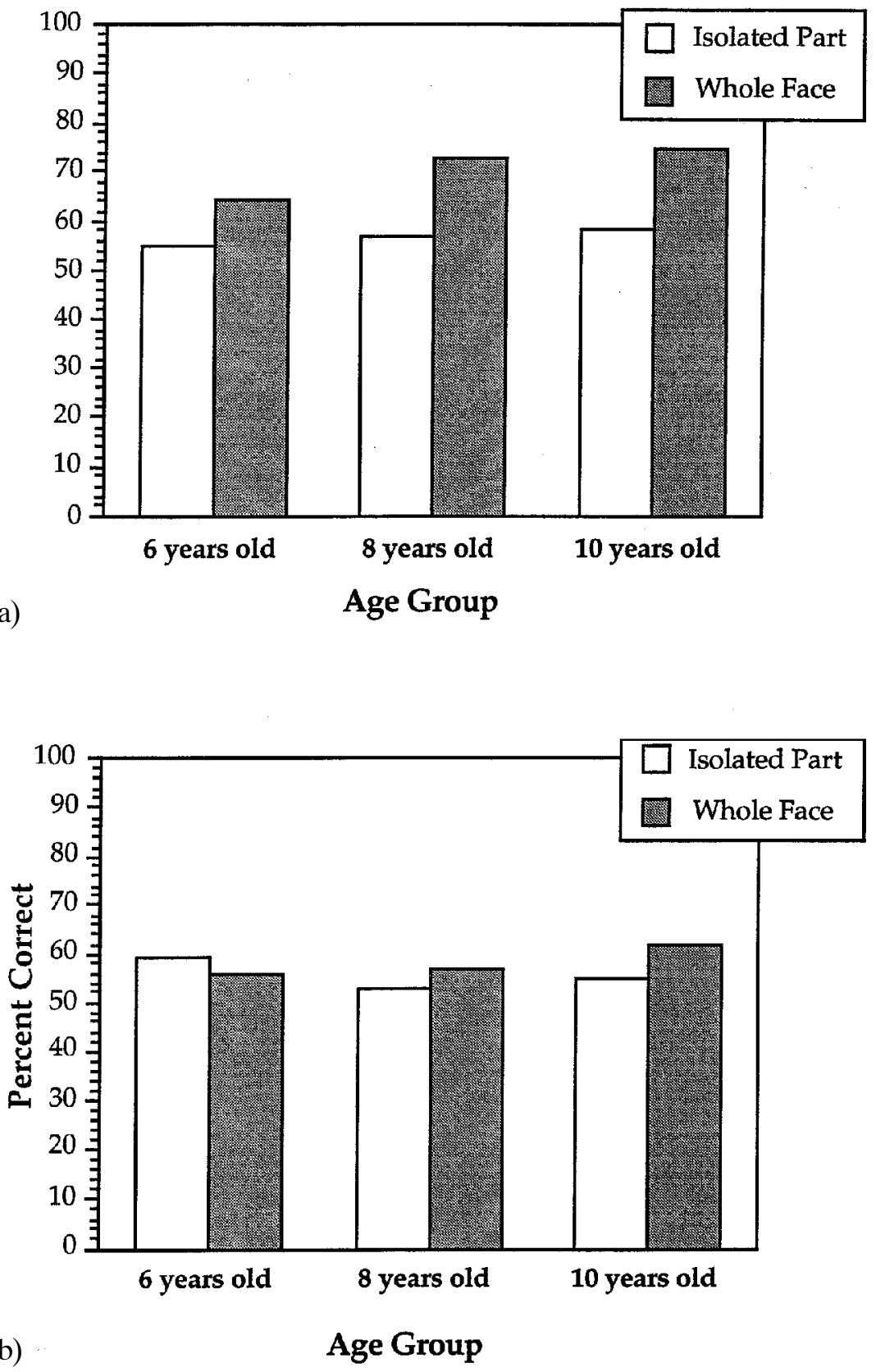

FIG. 5. Results of Experiment 2. (a) The percentage of correctly identified isolated-part and wholeface test items tested in their upright orientation as a function of age. (b) The percentage of correctly identified isolated-part and whole-face test items tested in their inverted orientation as a function of age. 
However, the single-process account still falls short of explaining what maturational or experiential factors might affect changes in the absolute level of holistic processing. Carey and Diamond (1994) have suggested that normbased coding might account for the age $\times$ inversion effect interaction. In a norm-based coding scheme, relational properties of a face (e.g. spatial distance between eyes and nose) are encoded relative to the prototypical or norm face in the population. As an individual acquires more experience with individual face exemplars, the norm representation better approximates the true population prototype. The more veridical norm representation can be used to resolve relational differences between highly similar faces, thereby leading to improved recognition of upright faces. Inversion, on the other hand, eliminates any expertise advantage by blocking norm-based encoding of relational properties (Bartlett \& Searcy, 1993; Rhodes, Brake, \& Atkinson, 1993; Tanaka \& Sengco, 1997). Thus, the norm-based model predicts that, whereas recognition of upright faces should improve with age, recognition of inverted faces should remain fairly constant across different groups. This is exactly the pattern of results that Carey and Diamond $(1977,1994)$ found in their development tests of the face inversion effect.

Two empirical studies are consistent with the norm-based coding account. First, Ellis (1992) found that 6- and 7-year-olds, unlike adults, do not recognize atypical faces better than typical faces. The absence of an atypical advantage indicates that young children have not yet honed their norm-based representation to the point where it would allow them to distinguish between typical and atypical faces. Thus, failing to encode the more distinctive properties of the atypical face, young children do not show a recognition advantage for atypical faces over typical faces. In another study, Tanaka and Farah (1991) found that adult participants did not demonstrate a larger inversion effect for the recognition of artificial dot patterns that shared a prototypical configuration relative to those patterns that did not. Because participants in this study received only limited exposure to the configural dot patterns, they did not have sufficient experience to generate a norm-based representation. In a sense, adults learning artificial dot patterns are analogous to 6-year-olds learning faces-both groups lack a norm representation that would facilitate the recognition of upright stimuli.

While we interpret our results as supporting the holistic approach to face processing, these findings are also consistent with the relational view of face recognition (Bartlett \& Searcy, 1993; Diamond \& Carey, 1986; Rhodes et al., 1993). According to the relational view, the spatial relations between facial features are as vital to face recognition processes as the features themselves. Although the holistic view acknowledges the importance of relational information in face processing, it does not treat featural and configural information as separable sources of information. Unfortunately, the holistic and relational hypotheses are not distinguishable in the present part-whole 
paradigm where both holistic and relational information are disrupted when a face part is tested in isolation. However, in a recent study, Tanaka and Sengco (1997) found that altering the featural relations of a face (i.e. increasing the inter-eye distance) impairs recognition of its eyes, nose and mouth features. The finding that configural and featural information are encoded together in the face representation is, therefore, supportive of the holistic approach to face recognition.

\section{REFERENCES}

Baenninger, M. (1994). The development of face recognition: Featural or configural processing? Journal of Experimental Child Psychology, 57, 377-396.

Bartlett, J.C., \& Searcy, J. (1993). Inversion and configuration of faces. Cognitive Psychology, 23, 281-316.

Carey, S., \& Diamond, R. (1977). From piecemeal to configurational representation of faces. Science, 195, 312-314.

Carey, S., \& Diamond, R. (1994). Are faces perceived as configurations more by adults than by children? Visual Cognition, 1, 253-274.

Carey, S., Diamond, R., \& Woods, B. (1980). The development of face recognition-a maturational component? Developmental Psychology, 16, 257-269.

Diamond, R, \& Carey, S. (1986). Why faces are and are not special: An effect of expertise. Journal of Experimental Psychology: General, 115, 107-117.

Ellis, H.D. (1992). The development of face processing skills. In V. Bruce, A. Cowey, A.W. Ellis, \& D.I. Perrett (Eds), Processing the facial image (pp. 105-111). Proceedings of a Royal Society Discussion Meeting, 9-10 July 1991. Oxford: Clarendon Press.

Farah, M.J., Drain, M., \& Tanaka, J.W. (1995). What causes the inversion effect? Journal of Experimental Psychology: Human Perception and Performance, 21, 628-634.

Flin, R.H. (1985). Development of face recognition: An encoding switch? British Journal of Psychology, 76, 123-134.

Johnson, M.H., \& Morton, J. (1991). Biology and cognitive development: The case of face recognition. Oxford: Blackwell.

Kemler Nelson, D.G. (1989). The nature and occurrence of holistic processing. In B.E. Shepp \& S. Ballesteros (Eds), Object perception: Structure and process (pp. 387-419). Hillsdale, NJ: Erlbaum.

Rhodes, G., Brake, S., \& Atkinson, A. (1993). What's lost in inverted faces? Cognition, 47, 25-57.

Saltz, E. \& Siegel, I.E. (1967). Concept overdiscrimination in children. Journal of Experimental Psychology, 73, 1-8.

Tanaka, J.W., \& Farah, M.J. (1991). Second-order relational properties and the inversion effect: Testing a theory of face perception. Perception and Psychophysics, 50, 367-372.

Tanaka, J.W., \& Farah, M.J. (1993). Parts and wholes in face recognition. Quarterly Journal of Experimental Psychology, 46A, 225-245.

Tanaka, J.W., \& Sengco, J.A. (1997). Features and their configuration in face recognition. Memory and Cognition, 25, 583-592.

Valentine, T. (1988). Upside-down faces: A review of the effect of inversion upon face recognition. British Journal of Psychology, 79, 471-491.

Yin, R.K. (1969). Looking at upside-down faces. Journal of Experimental Psychology, 81, 141-145. 
Young, A.W., Hellawell, D., \& Hay, D.C. (1987). Configurational information in face perception. Perception, 16, 747-759.

Manuscript received September 1996 Revised manuscript received September 1997 